\title{
A Metrology Approach to Uncertainty in Quantitative EDS Analyses
}

\author{
S.N. Prins, ${ }^{*}$ L.H. Adlem* and M.E. Lee** \\ * CSIR-National Metrology Laboratory, PO Box 395, Pretoria, 0001, South Africa \\ ** Centre for Electron Microscopy, University of the North, Sovenga, 0727, South Africa
}

Energy dispersive X-ray spectroscopy (EDS) is a routine non-destructive analytical tool for elemental analysis of materials on a micrometer scale, since elements can be detected from levels of about 0.1 wt. \%. However, since the final EDS results are based on the counting statistics, the spectrum processing, the model corrections to convert intensities to concentrations, the standards used and the operating conditions, the uncertainties associated with the results can be significant.

Uncertainty is the parameter associated with the result of a measurement that characterises the dispersion of values that could reasonably be attributed to the measurand [1]. Uncertainties are classified as Type A or Type B. A Type A uncertainty is determined by statistical analysis of a series of observations, while Type B uncertainties are based on scientific judgements, such as previous data and/or experience, manufacturer's specifications, uncertainties assigned to reference materials. Uncertainty is generally expressed as a standard deviation, and called the standard uncertainty. However, often a number of factors contribute to the total uncertainty of a measurement. These factors can be uncorrelated (independent) or correlated (interdependent). To combine uncorrelated uncertainties, they must all be written in the same units of measurements. Following the ISO GUM guide [1], the uncertainties are converted to variances, which can be summed linearly. This is then called the combined standard uncertainty.

The CIPM (Comité International des Poids et Mesures) requests that participants in international intercomparisons use the term 'combined standard uncertainty', $\mathrm{u}_{\mathrm{c}}(\mathrm{y})$ and that they denote the level of confidence of the uncertainty for commercial, industrial, accredited and regulatory use as an expanded uncertainty. The expanded uncertainty is obtained by multiplying the combined uncertainty with a coverage factor. The coverage factor is determined from the effective degrees of freedom associated with the measurement. Each uncertainty contributor has associated with it a certain number of degrees of freedom. The effective degrees of freedom are calculated from the individual contributions using the Welch-Satterthwaite formula [1].

EDS analyses falls into the measurement of amount of substance in the field of chemical metrology. Chemical metrology is a relative new field, in which surface and microanalyses are even newer. In preparation for ISO 17025 accreditation of the analytical techniques of the Surface and Microanalysis group at the CSIR-NML, we have started to identify and classify the sources of uncertainty in EDS analysis. As there are no official guidelines for uncertainty calculation of EDS results according to the CIPM guidelines, a number of processes have been initiated to monitor EDS performance and results in order to assign statistical uncertainties to the factors influencing EDS results.

A number of system performance monitoring procedures have been set up to statistically evaluate the influence of some of the EDS parameters. On a weekly basis, the Mn and C peak resolutions, the $\mathrm{Cr} \mathrm{K} \alpha$ and $\mathrm{Cu} \mathrm{K}: \mathrm{L}$ line ratios are monitored to determine the stability of the EDS system. The Mn K $\alpha$ peak is used to track spectral resolution, and should be similar to the 
value quoted by the EDS detector resolution specified by the manufacturer. The $\mathrm{C} \mathrm{K} \alpha$ peak resolution is used to monitor the detector's response at low voltages. The $\mathrm{Cr} \mathrm{K} \alpha$ is used to track the deviation (if any) of the peak due to Incomplete Charge Collection (ICC) caused by the detector dead layer. The value for the ICC should ideally be equal to $1.82 \mathrm{for} \mathrm{Cr} \mathrm{K} \alpha$. The $\mathrm{Cu} \mathrm{K}: \mathrm{L}$ line ratio gives information on the low energy efficiency of the window as well as the build up of contamination on the detector window.

Initially, spectra were acquired using short live times and low dead times, and this reflected in the results of the measurements. In Fig. 1, the Mn-peak resolution results are shown as a CUSUM chart. The initial results are scattered and shows significant detector fluctuation. However, after the procedure was changed to EDS acquisition conditions of 100 seconds livetime at $20 \mathrm{kV}$ and a 30\% dead time (approximately 2200 counts per second), the results became more consistent. This also confirmed that the statistical error in counting the X-rays is one of the biggest sources of uncertainty in EDS results.

In the second phase of the project, the influence of sample homogeneity and spectrum acquisition mode was evaluated using the NIST SRM 482 Gold-Copper standard. Spectra were acquired as a number of acquisitions on a single large area $\left(0.13 \mathrm{~mm}^{2}\right)$, a single acquisition of a number of small areas $\left(780 \mu \mathrm{m}^{2}\right)$, single acquisitions on a number of random points and points on fixed intervals on a line. The total integrated spectrum counts were \pm 220000 , with the net counts above the background for the $\mathrm{Cu} \mathrm{K \alpha} \pm 25000$ and $\mathrm{Au} \mathrm{M} \alpha$ \pm 18000 counts. Using standard statistical f and t-tests, it was determined that there were no significant differences between the precisions and accuracies of the four acquisition modes. However, when the t-test was used to validate the accuracy of the EDS method (comparing to the SRM), there were significant differences at both the 95\% confidence level and the $99 \%$ confidence level. Only the results of the random points at $99 \%$ (3 sigma) were acceptable.

As part of a three-year project, the factors that could influence EDS results will systematically be analysed and the uncertainties will be classified as Type A or Type B uncertainties. An uncertainty budget for EDS measurements in metrology will be proposed.

\section{References}

[1] Guide to the Expression of Uncertainty in Measurement, ISO, Geneva (1993).

[2] J.C. Russ, Fundamentals of Energy Dispersive X-ray Analysis, Butterworths (1984).

[3] The financial assistance of the DTI, South Africa is gratefully acknowledged.

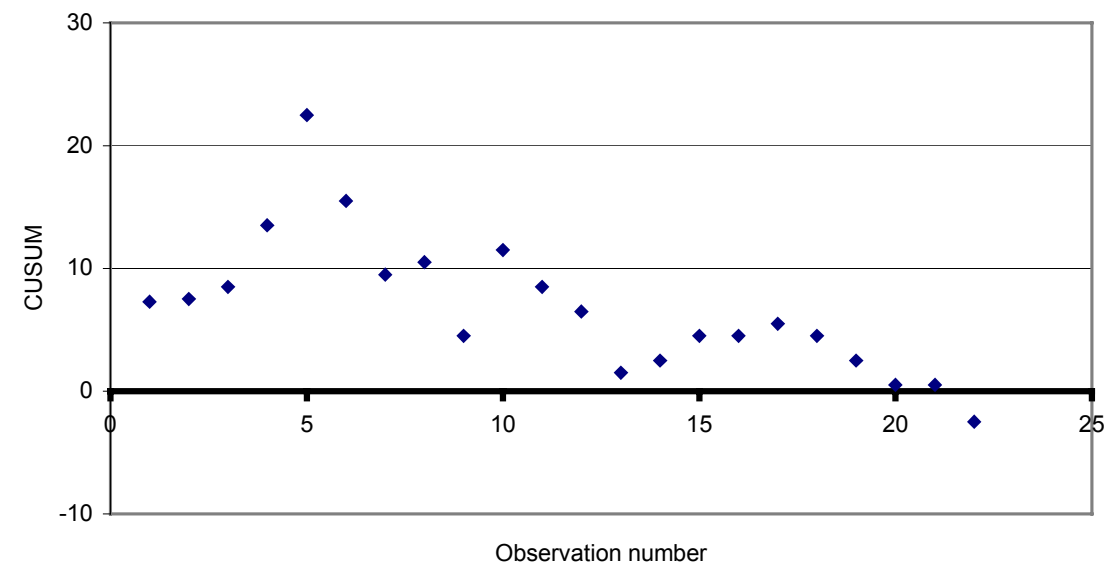

Figure 1. CUSUM chart tracking the Mn Ka resolution test. 